\title{
DOSSIER
}

\section{DÉVELOPPEMENT DE L'ENTRE-SOI COMMUNAUTAIRE DANS LES ESPACES RÉSIDENTIELS PÉRIURBAINS}

\author{
Jacques Teller*
}

Le texte aborde le développement de l'entre-soi communautaire dans les espaces périurbains. Sur base de l'exemple de la Région Wallonne, nous suggérons de replacer ce débat dans un cadre étendu afin de prendre en considération une évolution du rapport social à l'espace, qui se construit autour de territoires de vie en réseaux avec pour corollaire une perte de signification de l'environnement de proximité. Dans un tel contexte, la relation entre environnement et sécurité gagnerait à être réfléchie à l'échelle métropolitaine plutôt qu'à celle de quartiers, à l'image du mouvement opéré par les sciences environnementales.

\section{Introduction}

Il s'agit, dans le cadre de cet article, de s'interroger sur l'actualité d'une approche de la sécurité fondée sur le contrôle social de l'environnement résidentiel local, tel que préconisé par la théorie de l'espace défendable. Nous prendrons appui ici sur le cas de la Région Wallonne dont le territoire a connu au cours des dernières décennies une dynamique de périurbanisation assez profonde. L'accroissement inédit des mobilités quotidiennes y a amené le développement de ce que d'aucuns appellent des territoires en réseaux (Offner, Pumain, 1996; Ascher, 1995), au niveau de l'économie bien sûr, mais aussi des espaces de vie des ménages et des individus. Cette mobilité s'accompagne d'une liberté accrue dans les choix de localisation de certaines populations qui peuvent ainsi échapper aux contraintes de la proximité urbaine. Ce phénomène est particulièrement aigu dans les régions soumises à une forte désurbanisation et où l'environnement résidentiel local semble perdre une part de sa consistance en terme de vecteur d'intégration et de médiation sociale.

L'émergence des théories de l'espace défendable aux États-Unis s'inscrit, rappelons-le, dans la foulée de la critique des quartiers planifiés au cours des années 1970. Conçus selon les préceptes du mouvement d'architecture moderne, ces quartiers étaient organisés autour d'ensembles d'immeubles en hauteur et réservaient l'espace au sol à un statut public, sans restreindre leur usage aux seuls habitants de ces ensembles.

Les reproches adressés à ce modèle sont aujourd'hui bien connus. Le contrôle social des espaces publics y est rendu difficile en raison de la distance visuelle et spatiale entre habitations et espaces communs. Le caractère monofonctionnel des tours de logement, et en particulier l'absence d'animation des rez-de-chaussée, accentue encore cette absence de contrôle social. Enfin l'indéfinition même du statut des espaces ouverts (on parlait alors d'espaces «libres ») prête à des formes de territorialisation incompatibles avec leur usage

* LEMA - Université de Liège. 
collectif. La conjonction de ces différents phénomènes peut entraîner le développement d'un sentiment d'insécurité auprès des habitants de ces ensembles de logements, sentiment essentiellement lié à des phénomènes d'incivilité.

Ce diagnostic devait amener des chercheurs américains, emmenés par Oscar Newman, à défendre un modèle de développement alternatif des espaces résidentiels. Par espace défendable, on entend un environnement résidentiel dont les caractéristiques physiques, disposition des bâtiments et plan du site, fonctionnent de manière à permettre aux habitants eux-mêmes de jouer un rôle actif afin d'assurer leur propre sécurité (Newman, 1975). Ce modèle est orienté autour d'immeuble de 3 à 4 étages au plus et prévoit une animation fonctionnelle des rez-de-chaussée ainsi qu'une hiérarchisation claire du statut des espaces ouverts (figure 1). Dans ce modèle, la majorité de l'espace ouvert est sous le contrôle social constant de la communauté locale qui l'habite.

Figure 1: Mode d'organisation urbaine préconisée par Newman pour renforcer le contrôle social des habitants à travers une hiérarchisation des espaces publics et semi-publics (Source: Newman, 1975).

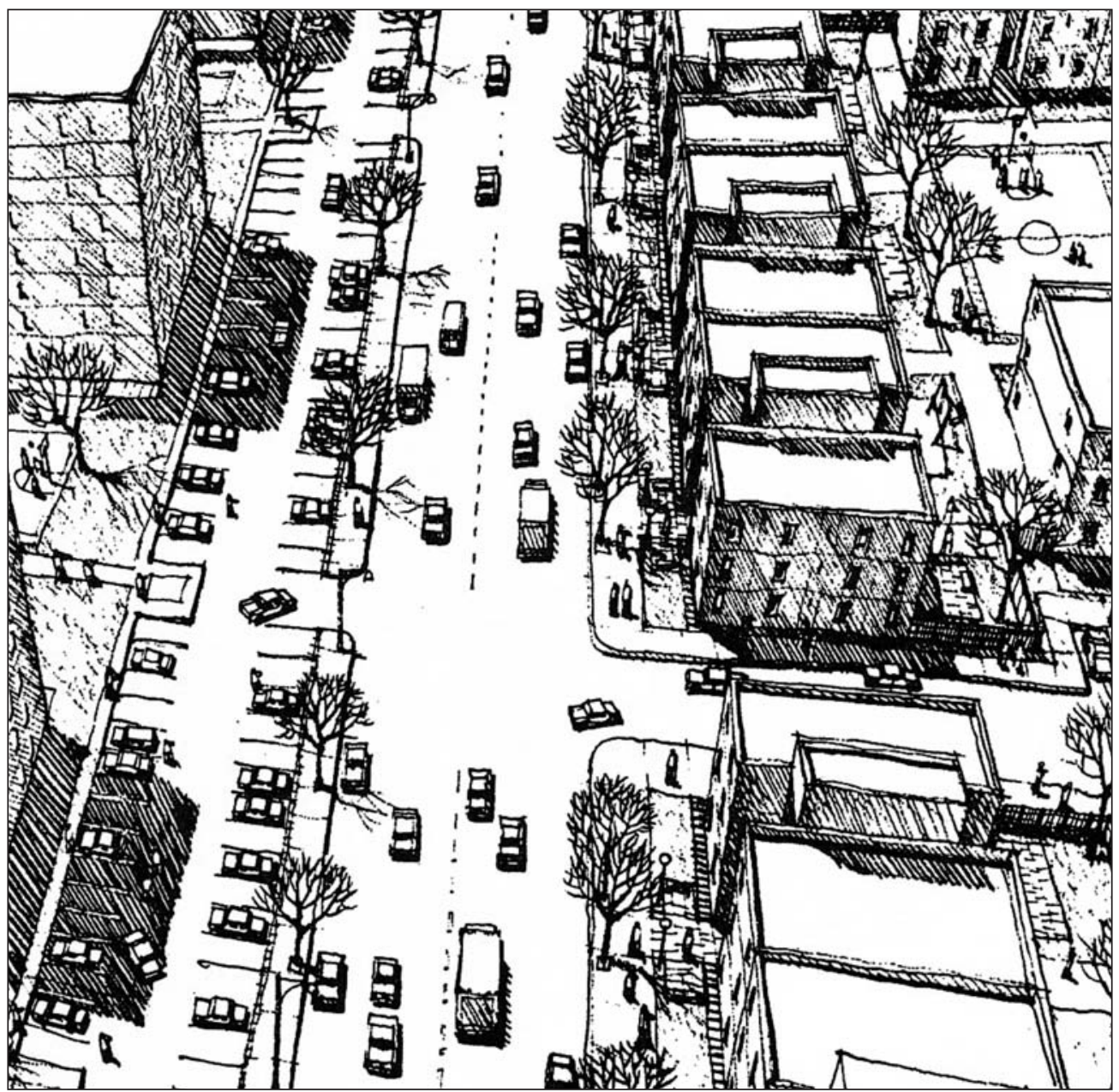


Cette démarche ouvrait la porte à une nouvelle conception de la sécurité en milieu urbain. Celle-ci voit dans la gestion de l'environnement physique un moyen efficace de réguler les rapports entre groupes sociaux et par là, de renforcer le sentiment de sécurité des habitants.

Nous souhaitons, dans le cadre de cet article, interroger l'actualité de ce modèle au regard des dynamiques de périurbanisation actuelles. Le rapport des individus à l'espace s'est de fait profondément transformé au cours des dernières décennies pour se structurer de manière croissante autour de territoires de vie en réseau. Cette évolution du rapport à l'espace est concomitante de la recherche d'entre-soi résidentiel dans un certain nombre de lotissements périurbains. Ce type de développement est renforcé, en Région Wallonne, par un mécanisme de financement communal qui privilégie assez nettement les localisations périphériques par rapport aux localisations centrales. Dans ce contexte, des politiques sécuritaires trop exclusivement centrées sur le contrôle social de l'espace résidentiel local sont de nature à renforcer la fragmentation de l'espace et, par là, à peser sur la cohésion sociale.

\section{Des territoires de vie en réseau}

La mobilité accrue des citoyens, tant dans le cadre de leur vie professionnelle que privée (achats quotidiens, activités récréatives, déplacements domicile-école, etc.) est aujourd'hui bien documentée. Si elle se manifeste de manière différenciée à l'échelle de l'ensemble du territoire national, elle est particulièrement marquée dans les grandes agglomérations et dans les territoires fortement urbanisés comme la Belgique. En raison du niveau d'équipement de ces agglomérations, on assiste à l'avènement d'un espace quasi isotrope, où, à l'intérieur d'une enveloppe assez lâche, toutes les positions sont équivalentes et le statut des nœuds centraux est fortement remis en cause.

L'insertion dans des réseaux apparaît comme la clé de l'intégration sociale et économique. Ce constat, pour trivial qu'il puisse paraître dans le champ socio-économique, marque profondément la relation des habitants à leur espace de vie quotidien. Ceux-ci donnent plus d'importance aux axes, nœuds et réseaux urbains qu'à leur quartier, ce qui remet profondément en question le rôle et le statut de l'environnement local comme espace d'insertion communautaire et de contrôle social.

Le hiatus grandissant entre quartier et espace de vie est particulièrement patent auprès des jeunes. Une enquête menée en 1998 auprès d'élèves de 13 à 14 ans illustre ce décalage (Teller, Vauchel, 2001). L'enquête a été menée dans un établissement scolaire situé sur la rive gauche de Huy (Belgique). Cette école se trouve au cœur d'un espace urbain assez homogène, ceinturé par une rivière, une voie pénétrante et une ligne de chemins de fer, à savoir les conditions «idéales» pour que cet espace puisse être appréhendé comme un quartier facilement identifiable. Il ressort des cartes mentales réalisées auprès des élèves de l'école qu'il n'existe aucun consensus quant à la définition des limites du quartier. La définition qu'ils donnent de leur espace de proximité est tantôt extrêmement étroite, réduite à quelques rues, tantôt extrêmement lâche, englobant l'ensemble du versant gauche de la vallée. Par ailleurs, les lieux qu'ils fréquentent avec leurs amis sont presque systématiquement localisés à l'extérieur du quartier, dans des endroits aussi diversifiés que le centre-ville, les berges du fleuve ou une zone de logements sociaux situés au nord de l'entité. Dans un tel contexte, quel est l'environnement local pertinent pour renforcer leur sécurité ? Quel rôle peut encore jouer le voisinage direct dans le contrôle social? 
Il n'est pas surprenant que les questions de sécurité se soient aujourd'hui déplacées depuis les espaces de résidence vers d'autres lieux stratégiques du territoire urbain: les nœuds et axes de communication. Si les agressions dans les transports en commun font aujourd'hui les grands titres de la presse, ce n'est pas seulement en raison de leur éventuelle recrudescence (à démontrer) qu'en raison de la sensibilité des citoyens à cette ressource vitale qu'est la mobilité dans un environnement urbain de plus en plus éclaté en un patchwork qui ne tient que par la solidité des liaisons entre ses différentes composantes. Les prémisses du plaidoyer de Newman en faveur d'espaces défendables semblent ainsi quelque peu invalidées par l'évolution récente du système urbain dans la mesure où l'enjeu sécuritaire s'est déplacé depuis l'espace de proximité vers les réseaux de déplacement à plus ou moins grande échelle.

\section{Le développement de l'entre-soi}

Au moment où la mixité sociale est préconisée dans les quartiers dits en difficulté, la localisation des ménages à revenus moyens et aisés semble toujours plus guidée par des choix relatifs à l'accessibilité aux infrastructures, aux services urbains, à l'espace vert, mais aussi au profil social des espaces résidentiels. Nous distinguerons ce phénomène des communautés encloses, qui ne sont qu'une de ses matérialisations possibles. Une communauté enclose est, au sens strict du terme, caractérisée par une clôture physique de son périmètre, une restriction des entrées et sorties ainsi que des règles contractuelles de gouvernance territoriale qui portent sur l'aménagement du domaine privé, sur le profil des candidats à la résidence ainsi que les charges collectives qui leur sont imposées. Ce type d'environnement constitue aujourd'hui un «produit» immobilier à part entière, mis sur le marché par des promoteurs qui prennent en charge la construction de l'ensemble du lotissement et des services qui lui sont associés (club house, tennis, piscine, etc.).

Ce type de développement est plutôt rare en Belgique. Le marché immobilier y est en effet aux antipodes du modèle de promotion groupée, caractéristique du monde anglosaxon (figure 2). La Belgique est le pays européen où le taux d'auto-promotion est le plus important en Europe: les propriétaires sont en général maîtres d'ouvrage de leur propre habitation. Le modèle le plus courant de production du logement repose sur l'achat d'un terrain vierge par le candidat bâtisseur, qui choisit et finance lui-même l'architecte et les entreprises de constructions qu'il souhaite. Le développement d'ensembles d'habitations homogènes par un promoteur immobilier y est nettement moins fréquent que dans les autres marchés nationaux, si ce n'est dans le cadre du logement social.

Si elle constitue un frein puissant à la constitution de communautés encloses sur le modèle américain, cette caractéristique du marché immobilier belge ne constitue nullement un frein au développement de l'entre-soi résidentiel. Derrière un vernis d'individualisation prononcée, liée à l'adaptation de l'habitat aux goûts et caractéristiques de chaque ménage, on observe une diffusion massive de la «villa quatre façades » dans les ensembles résidentiels. La banalisation du paysage périurbain est liée à une certaine homogénéité des attentes et des modes de vie des candidats bâtisseurs : dégagement vis-à-vis des voisins et de l'espace public, présence d'un garage accessible depuis la voirie, dissociation verticale des lieux de vie et de repos, protection de l'intimité depuis l'espace public et larges ouvertures sur le jardin situé à l'arrière de l'habitation.

Tous ces éléments tendent à produire un milieu périurbain assez homogène, sans qu'il n'ait été programmé par un quelconque promoteur. Le modèle de base de la maison quatre 
Figure 2: Variation du taux d'autopromotion du logement en Europe. On y constate que l'autopromotion est beaucoup plus développée en Belgique que dans d'autres pays européens, en particulier la GrandeBretagne (Source: Duncan, Rowe, 1993).

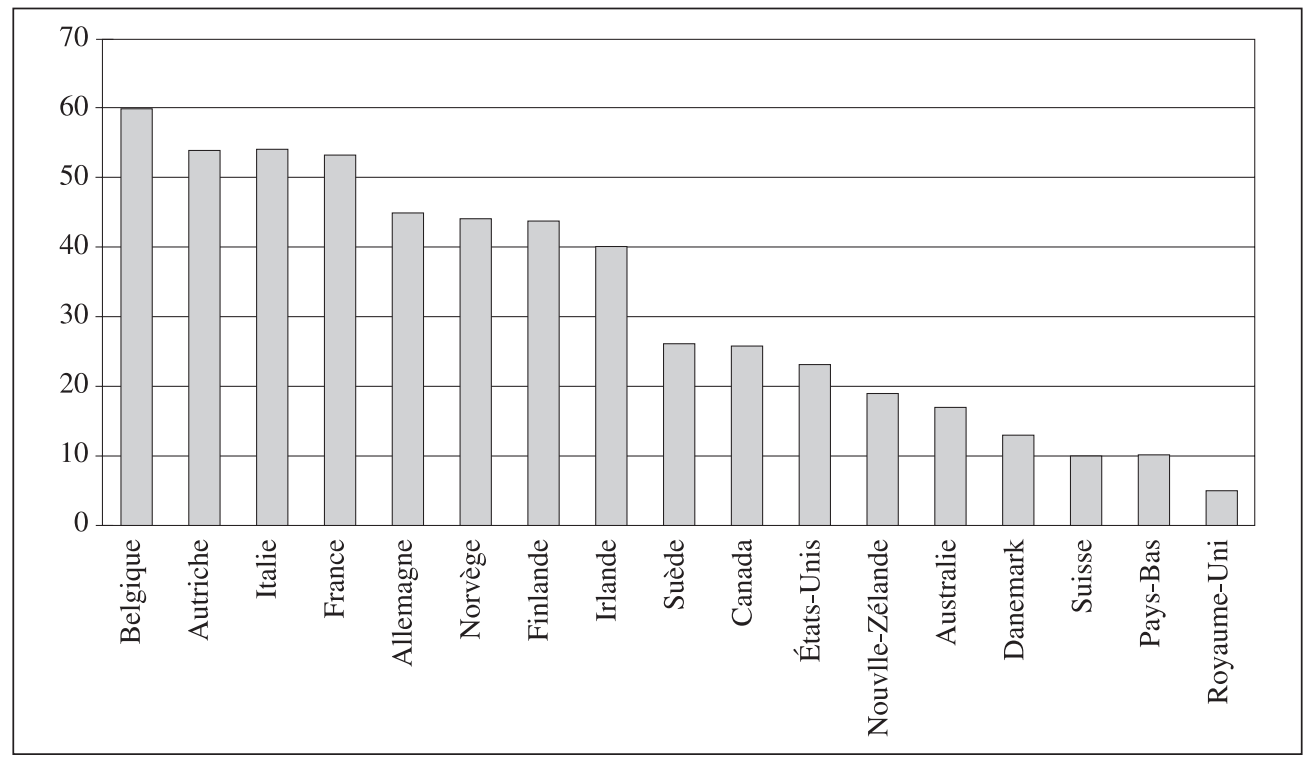

façades entourées d'un jardin offre toutefois assez de latitude pour permettre une différenciation assez nette entre lotissements aisés et modestes. Par ailleurs la conquête de l'espace périurbain s'effectuant par vagues successives, on assiste à une segmentation de l'espace périurbain par tranches d'âge selon la date d'ouverture des lotissements. Si le seul jeu de l'offre foncière de parcelles individuelles suffit à induire un regroupement par groupes sociaux, celui-ci est encore renforcé par les contraintes réglementaires publiques et/ou privées. Ces contraintes portent sur la taille maximale des parcelles, le type d'urbanisation autorisé dans les zones à bâtir (pavillonnaire ou groupé), voire plus classiquement la résistance des communes périphériques à favoriser une densification des zones qui restent urbanisables. Il n'est nul besoin de barrière ou de dispositifs d'isolement pour marquer les frontières symboliques entre différentes communautés, même si la ségrégation n'est jamais aussi accomplie que dans les communautés encloses en raison même de son caractère spontané et du jeu des achats et reventes d'habitations existantes.

La fragmentation urbaine et l'entre-soi résidentiel ne sont en rien une exclusivité des communautés encloses; ils peuvent être plus ou moins choisis ou imposés selon les catégories sociales considérées. Dans tous les cas, ils semblent moins répondre à une véritable insécurité ou à une hypothétique «peur de l'autre» qu'au souhait de vivre entre personnes de même qualité et partageant un ensemble de valeurs communes (Chevalier, Carballo, 2004). Un certain nombre d'éléments de mobilier urbain, tels que les casse-vitesse, chicanes ou barrières végétales peuvent éventuellement compléter le dispositif et agir de manière dissuasive vis-à-vis d'éventuels «intrus », même s'ils ont un caractère perméable et sont aménagés par les autorités publiques. Ils manifestent, de façon plus ou moins explicite, une volonté de mise à distance vis-à-vis de nuisances de voisinage jugées indésirables: trafic automobile, incivilités, regroupements de jeunes dans l'espace public, etc. 
Le milieu périurbain constitue un terreau très favorable au développement de l'entresoi résidentiel. La densité très faible de ces environnements, les limites que constituent les voiries à grand trafic et la présence de nombreuses poches agricoles ou naturelles intercalaires ne peuvent que renforcer la ségrégation entre groupes sociaux et culturels, même si celle-ci est largement due à des facteurs socio-économiques comme l'accès au marché immobilier. Les centres-villes ne sont pas épargnés par ce phénomène, mais ils ont connu une hybridation beaucoup plus forte au cours des dernières années et ils sont les premiers à souffrir de l'hémorragie des classes moyennes et aisées.

\section{Périurbanisation et cohésion sociale}

Il n'existe pas de relation simple, univoque entre forme urbaine et rapports sociaux. Une même forme urbaine peut accueillir au cours du temps différents types d'occupation et des profils de population et d'usages assez variés. Reste toutefois que l'on peut défendre que certains environnements urbains peuvent être plus ou moins favorables à certains types d'appropriation et de relations sociales. On peut ainsi affirmer que le milieu périurbain est à la fois le produit et le vecteur d'une mise à l'écart vis-à-vis de ce qui forme traditionnellement la trame de l'espace public conventionnel: le côtoiement de personnes inconnues, le frottement entre populations issues de classes sociales différentes, la rencontre des différents groupes culturels que rassemble inévitablement la ville contemporaine.

Il permet, plutôt qu'il n'induit, une plus grande distance sociale entre différents groupes partageant un certain nombre de valeurs et des ressources sociales, économiques, culturelles. D'aucuns défendent aujourd'hui que cette ségrégation n'est pas problématique, dans la mesure où elle est liée de manière intemporelle au fait urbain. Par ailleurs, les espaces de friction entre groupes sociaux et culturels n'auraient pas disparu du paysage urbain, mais se seraient plutôt déplacés vers d'autres lieux de sociabilité tels que les ensembles commerciaux, les gares et les complexes de loisir (Ascher, 1995). Cette critique est pertinente au regard de l'émergence de territoires en réseau que nous avons évoquée, mais elle n'épuise pas pour autant la question. En effet, ces lieux d'activité sont généralement de statut privé et leur accès est bien souvent restreint, ne fut-ce qu'en raison de contraintes de déplacement et de ressources financières quand ce n'est pas tout simplement par des mesures de gardiennage. On assiste de ce fait à une dichotomie croissante entre des populations bien connectées, ayant accès à ces nouvelles infrastructures urbaines et celles qui sont contraintes de recourir à des services de proximité, dont la qualité et la diversité souffrent de la perte d'une part de leur clientèle.

À ce titre, alors que le territoire de la ville est traditionnellement le lieu de rencontre et de régulation entre des intérêts divergents, on constate aujourd'hui que les conflits entre différents groupes sociaux ne font plus l'objet d'une médiation quotidienne. L'existence même d'importantes poches de pauvreté au cœur des agglomérations urbaines ne pose pas de problème pour un certain nombre d'habitants de la périphérie dans la mesure où ils ont peu de chance de les côtoyer au jour le jour.

La situation des villes belges est assez différente de celle des villes françaises à cet égard, dans la mesure où l'on assiste à une inversion du gradient de pauvreté depuis le centre vers la périphérie (figure 3). La fuite des classes moyennes et aisées vers la périphérie a ainsi amené une concentration de ménages et d'individus à faibles revenus au centre-ville. 
Figure 3: Évolution des revenus des ménages dans la région bruxelloise. On discerne très clairement la concentration de ménages à revenus faibles au centre-ville et à revenus élevés dans les communes périphériques. L'écart entre ces deux pôles s'est très nettement accentué au cours de la période 1985-2000 (Source Boudry et al., 2004).

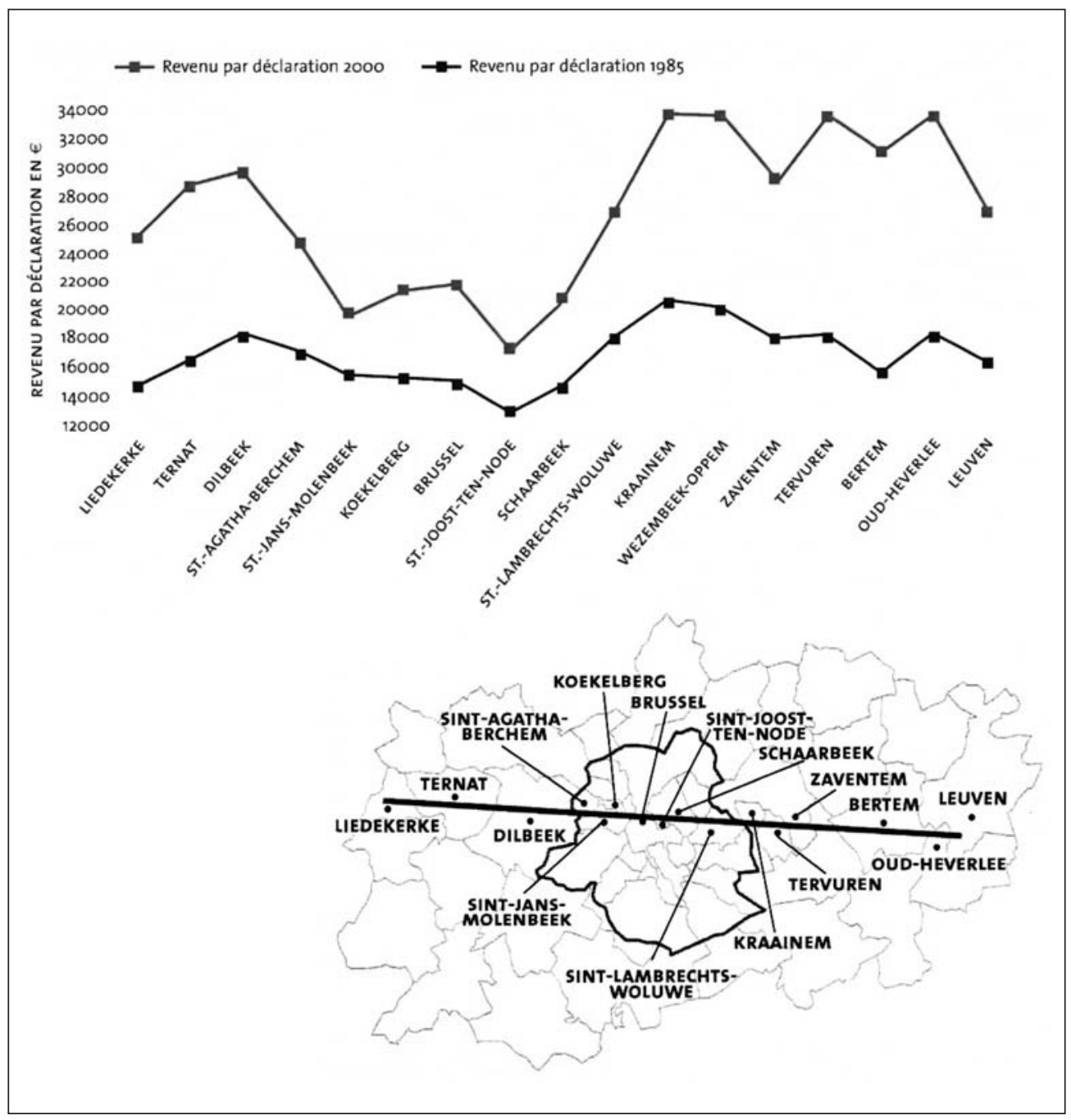

Cette tendance ne fait que s'amplifier au cours du temps dans la mesure où, en Région Wallonne, une fraction croissante des rentrées communales est liée à la part locale de l'impôt sur le revenu des personnes physiques (figure 4). Les communes centrales doivent dès lors assumer des charges croissantes en matière d'aide sociale, sans disposer des rentrées que pourraient occasionner la présence de ménages à revenus élevés sur leur territoire. Et elles ne peuvent pas investir dans de nouveaux équipements (espaces publics, espaces verts, loisirs) et leur entretien susceptibles d'attirer des ménages à revenus élevés. Une fois le mouvement amorcé, les communes périphériques se retrouvent alors en position privilégiée pour attirer des populations aisées et de nouveaux services commerciaux et culturels, ce qui ne fait qu'appauvrir les centres. 
Figure 4: Évolution comparée des dotations et des recettes fiscales des communes belges depuis la fusion des communes - budgets communaux en millions d'euros de 2000 (Source: Dexia Banque).

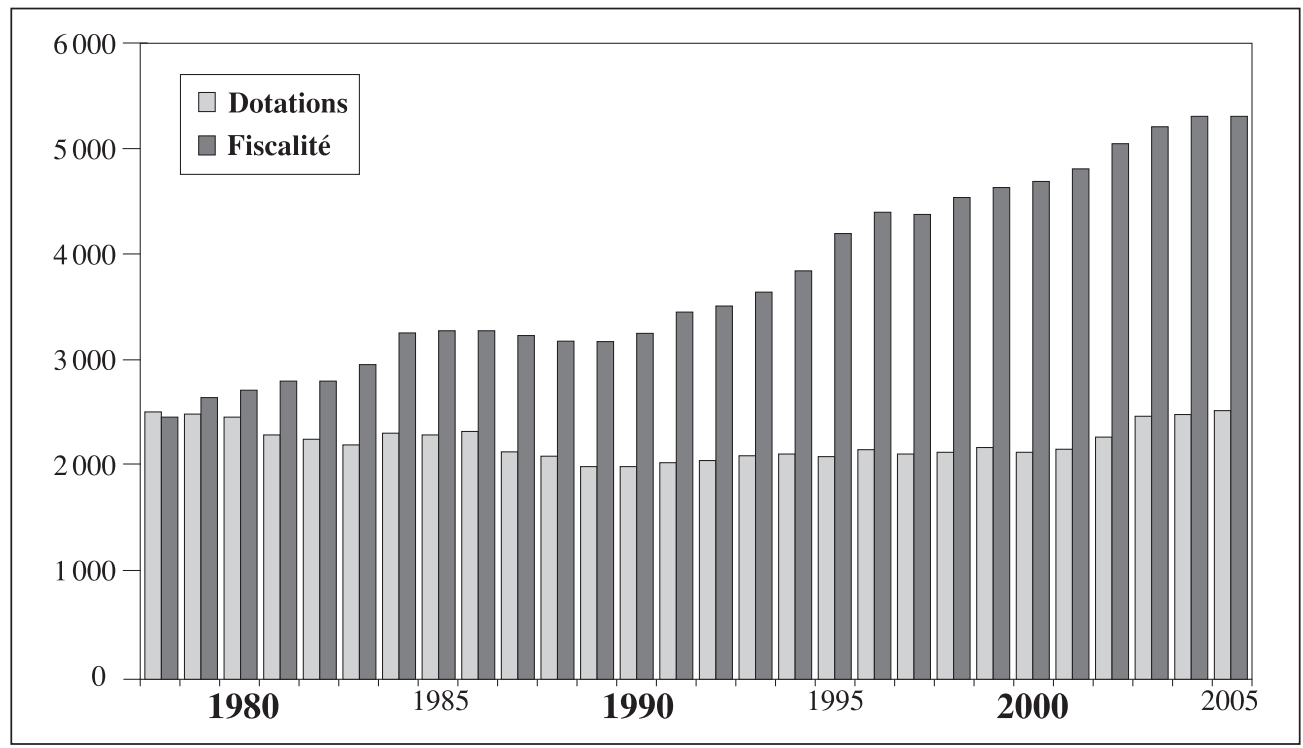

On se retrouve dès lors face à une situation où, en l'absence de véritables communautés encloses, le simple jeu de l'offre immobilière et de la compétition entre communes pour attirer des populations aisées suffit à produire une ségrégation assez marquée entre différents groupes sociaux. En terme de sécurité, ceci se traduit par une nette dévalorisation de l'image de certains centres-villes, associés à des actes d'incivilité et d'insécurité, ce qui contribue à renforcer leur déclin. On voit dans ce contexte qu'une action trop strictement orientée vers l'environnement local est inadaptée tant le phénomène d'insécurité est lié à des dynamiques urbaines qui se développent à l'échelle métropolitaine plutôt que locale et engagent des logiques de redistribution et de solidarité entre différentes communes qui paraissent aujourd'hui déficientes.

\section{Conclusions}

Les questions de sécurité et de cohésion sociale, si elles doivent s'envisager de manière conjointe, gagneraient à être posées à l'échelle des régions urbaines dans leur ensemble. L'émergence de territoires de vie en réseau, les nouvelles formes de ségrégation urbaine, la relative fragilité des nœuds et axes de liaisons qui permettent l'interconnexion du système urbain, tous ces facteurs, que nous avons évoqués au cours de cet article, concourent à asseoir cette conclusion. Dans le domaine environnemental, des analyses éco-systémiques de la ville ont amené à formuler des scénarios alternatifs en matière d'aménagement de l'espace urbain. Celles-ci sont déclinées en différentes formules, mais toutes reposent sur l'idée d'un développement polycentrique, dans lequel le milieu urbain n'est plus considéré comme partie du problème de la ville centre, mais plutôt comme partie intégrante et indispensable de son évolution (Urban Task Force, 1999). 
Organisés autour de couloirs de développement, bien desservis par les transports en commun, ces scénarios proposent des modèles fort proches de ceux qui étaient défendus par les théories de l'espace défendable pour ce qui est de l'aménagement de nœuds périurbains denses. Il y a là un espace de dialogue possible entre politiques sociales et environnementales, qui pourraient trouver dans le champ de l'espace périurbain un certain nombre de points de convergence. Le risque de voir se renforcer le décrochage entre centre et périphérie, ainsi qu’entre différents espaces périurbains, n’est toutefois pas négligeable. Il pourrait alors entraîner la consolidation de véritables communautés encloses, établies sur base des lotissements actuels. Le recours au gardiennage privé et les mesures d'isolement par des barrières que l'on n'observe encore que de façon assez sporadique ne seraient alors que les prémisses d'un phénomène plus préoccupant.

\author{
Jacques Teller \\ LEMA \\ Université de Liège \\ 3, boulevard du Rectorat \\ 4000 Liège \\ Belgique
}

\title{
BIBLIOGRAPHIE
}

ASCHER F., 1995, Métapolis, ou, L'avenir des villes, Paris, éditions Odile Jacob.

BOUDRY L., CABUS P., CORIJN E., DE RYNCK F., KESTELOOT C., LOECKX A., 2004, Livre blanc: Le siècle de la ville: de la république urbaine et de la ville trame, ministère de la Communauté flamande.

CHARMES E., 2006, La vie périurbaine face à la menace des gated communities, Paris, L'Harmattan, col. Villes et Territoires.

CHEVALIER J., CARBALLO C., 2004, Fermetures résidentielles et quête de l'entre-soi, entre Nord et Sud des Amériques, L'espace géographique, 4, 325-335.

DUBOIS O., 2008, Financement des communes et développement territorial durable en Région wallonne: quelles contraintes pour les communes?, Union des Villes et Communes, Rapport interne.

DUNCAN S.S., ROWE A., 1993, Self-provided Housing: «The first World's Hidden Housing Arm», Urban Studies, 30, 8, 1331-1354.

HALLEUX J.-M., LAMBOTTE J.-M., BRÜCK L., BEAUJEAN B., 2003, Production de l'urbanisation et infrastructures de viabilisation. Les coûts de la désurbanisation en Wallonie, Ruimte \& Planning, 23, 3, 124-137.

NEWMAN O., 1975, Design Guidelines for Creating Defensible Spaces, U.S. Department of Justice, U.S. Government Printing Office.

OFFNER J.-M., PUMAIN D., 1996, Réseaux et territoires. Significations croisées, Paris, éditions de l'Aube.

TELLER J., VAUCHEL B., 2001, Approche spatiale de l'exclusion, in BAWIN-LEGROS B., STASSEN J.-F., L'exclusion et l'insécurité d'existence en milieu urbain, éd. de l'Université de Liège, 123-149.

URBAN TASK FORCE, 1999, Towards an Urban Renaissance, E \& FN Spon.

\section{Summary}

The article looks at the development of community in suburban neighbourhoods. Considering the Walloon region as an example, we suggest that the debate needs to be resitu- 
ated and developed in order to take into consideration the evolution of the ways in which people relate socially to space, constructed through the ways in which people live their lives at work etc. with as a corollary a loss of significance for the near neighbourhood. In this context, the relationship between environment and attained security has been reflected at the city level more than at the neighbourhood level, in a similar way to that envisaged by environmental science.

\section{Zusammenfassung}

Der Text behandelt die Entwicklung homogener Gemeinschaften in Vorstädten. Auf der Grundlage des Beispiels der Region Wallonien wird vorgeschlagen, die Debatte in einen größeren Rahmen einzubetten und dabei die Evolution der sozialen Beziehungen zum Raum einzubeziehen. Dieser Beziehung konstruiert sich um ein Leben in Netzwerken mit der Folge, dass die unmittelbare Umwelt an Bedeutung verliert. In diesem Kontext sollte der Zusammenhang zwischen Umwelt und Sicherheit eher auf der Ebene der Stadt und weniger auf der Ebene von Stadtvierteln diskutiert werden.

\section{Sumario}

Este artículo aborda el desarrollo de las relaciones comunitarias en los espacios periurbanos. Tomando como ejemplo la región Valona, se sugiere ubicar este debate en un marco amplio que permita tomar en consideración la evolución de la relación social al espacio. Esta se construye alrededor de unos territorios de vida en red, lo que tiene como corolario la perdida de significación del entorno próximo. En ese contexto, sería preferible reflexionar sobre la relación entre entorno y seguridad a escala metropolitana en lugar de hacerlo a escala de los barrios, tomando ejemplo del movimiento que se ha operado en las ciencias del medio ambiente. 